radiating areas are traversed in equal times. The output of the microphone is amplified and applied to an analyser to determine the sound intensity in different frequency bands. A meter reading gives the average intensity of the sound in a selected band : and multiplication by a factor, involving the area of a hemisphere the radius of which is the length of microphone arm, gives the total power radiated in that particular band. This integrator measures sound outputs in about one fiftieth of the time previously required to make separate observations at many points about the source. It has been used extensively in developing telephone set housings.

\section{Electrical Demonstration Equipment}

IN the Bell Laboratories Record of July a description is given by C. D. Hanscom of a variety of equipment which has recently been assembled by the Bell System for use in public lectures. One of the most unusual demonstrations is a Rochelle salt crystal which flashes a neon lamp when hit with a gavel. This illustrates how a change in mechanical dimensions caused by the blow generates momentary voltages of considerable magnitude by the piezoelectric effect. A bar of steel (a permanent magnet) floating in mid-air demonstrates the power of modern magnets. A permanent magnet concealed in the base of the apparatus repels the bar, holding it up against the force of gravity ; a full packet of cigarettes can be supported in addition to the bar. There are also in the collection permalloy rods which are so permeable that they are magnetized by the earth's field, when held pointing north at or near the angle of declination. This is demonstrated by their ability to attract and hold short pieces of permalloy tape.

Decreased size of loading coils, made possible by research on magnetic alloys, is illustrated by a display board on which are mounted a coil with an iron-dust core, a much smaller coil of equal efficiency with a permalloy core, and a still smaller one with the same electrical characteristic, the core of which is molybdenum permalloy. Samples of the 2121-pair cable for exchange areas are included; also a piece of the Minneapolis-Stevens Point coaxial cable which transmits frequencies of several million vibrations per second. A replica of Bell's original telephone is usually included with the exhibits.

\section{Photography in Stellar Astronomy}

A RADio talk by the late Dr. Annie J. Cannon, entitled "The Story of Starlight", delivered on January 18 last, from Harvard Observatory, appears in the Telescope of May-June. A short description is given of the developments in spectroscopy since 1666 when Newton bought a crude prism at a country fair, "to try therewith the phenomena of colour". It is remarkable that two hundred years elapsed before Newton's work was carried to fruition, but when the potentialities in the study of spectra were realized, there was joy in being an astronomer. As Sir William Huggins remarked, "Those were the days when there was something worth while to do in astronomy". Dr. Cannon gives a brief account of photographic developments with special reference to the work of Harvard, where there are half a million negatives, which may be likened to a library of first and only editions, the whole forming the sole record of events observed in the stellar universe during the last half-century. The brief survey includes the important discovery of Miss Leavitt on the relation between the period of pulsation of a Cepheid variable star and its candle-power-a discovery which provides the data for determining the distances of these stars. This radio talk will be read with interest by the amateur astronomer.

\section{Nature Study for Evacuees}

THE Universities' Federation for Animal Welfare (U.F.A.W.) has issued two further useful and informative lectures for delivery to evacuee children, namely "British Snakes and Lizards", by Dr. R. C. Blackie, curator of Exeter Museum, and "Frogs and Toads", by E. M. Stevenson, lecturer in biology, University College, Exeter. The lectures are printed as brochures and accompanied by photographic plates for illustration and will help to solve a very pressing problem with many town teachers inexperienced of the countryside where they are now evacuated with their inquiring pupils. In a similar way, the various branches of the Workers' Educational Association have included nature study, biology, botany and kindred subjects in their programme of lecture courses for the coming winter. The West Lancashire and Cheshire Branch of the W.E.A., for example, is arranging nature study courses this winter at the University of Liverpool, Neston Library, Runcorn Technical Institute, Southport Technical Institute and probably Maghull Library, with a special appeal to teachers, and biology classes at the University and some of the branch towns. Attention has been given to nature study at the large Colomendey school camp, North Wales, but in most parts of the country considerable help in this subject is still required by town teachers in care of evacuees but handicapped by the limitations of their own experience of field natural history, which differs so radically from laboratory biology. A "Junior Naturalists' Society" has been formed. by F. Stodart at Longfield, Kent, and much help is being given by local branches of the British Empire Naturalists' Association.

\section{Bug Infestation}

In his latest annual report Sir Alexander Macgregor, medical officer of health for Glasgow, states that the total number of houses in that city in which bed bugs were found in 1939 was 309 , or $2 \cdot 1$ per cent, as compared with $3 \cdot 1$ per cent in 1938. In 79 houses, or 0.5 per cent only, a "trace" of bed bugs was found, as compared with 0.9 per cent in 1938. In this group only old hatched eggs were found, but no living bugs or eggs were detected in beds or furniture, pictures or household belongings. In 62 houses, or 0.4 per cent compared with 0.5 per cent in 1938 , a medium degree of infestation was found, that is, living bugs or eggs were present, but not in the 